\title{
EQUIVALENT NORMS IN SOME SPACES OF ANALYTIC FUNCTIONS AND THE UNCERTAINTY PRINCIPLE
}

\author{
BORIS PANEAH \\ Department of Mathematics, Technion \\ 32000, Haifa, Israel
}

\begin{abstract}
The main object of this work is to describe such weight functions $w(t)$ that for all elements $f \in L_{p, \Omega}$ the estimate $\|w f\|_{p} \geq \mathrm{K}(\Omega)\|f\|_{p}$ is valid with a constant $\mathrm{K}(\Omega)$, which does not depend on $f$ and it grows to infinity when the domain $\Omega$ shrinks, i.e. deforms into a lower dimensional convex set $\Omega_{\infty}$. In one-dimensional case means that $\mathrm{K}(\sigma):=\mathrm{K}\left(\Omega_{\sigma}\right) \rightarrow \infty$ as $\sigma \rightarrow 0$. It should be noted that in the framework of the signal transmission problem such estimates describe a signal's behavior under the influence of detection and amplification. This work contains some results of the above-mentioned type which I presented at the Banach Centre in the Summer of 1994. Some of these results had been published earlier, some are new ones.
\end{abstract}

Introduction. Uncertainty principle in Fourier analysis asserts that the more a function $f$ is concentrated the more its Fourier transform $F$ will be spread out. The corresponding nontrivial relations between $f$ and $F$ admit adequate physical interpretations, for instance in the framework of the signal transmission problem, in which the Fourier transform $F(\xi)$ of a signal $f(t)$ is interpreted as a bandwidth. From the physical point of view it is very natural to consider signals of $f(t)$ with compact supported bandwidths $F(\xi)$. Then the function $f(t)$ itself can be extended into the complex space $\mathbb{C}^{1}$ as an entire function of exponential type. And this is exactly the class of functions we deal with in the course of the paper. More exactly, let $f$ be a function on $\mathbb{R}^{n}$ and $F$ its Fourier transform defined by

$$
F(\xi)=(2 \pi)^{-n / 2} \int f(t) e^{-i<t, \xi>} d t
$$

where $t=\left(t_{1}, t_{2}, \ldots, t_{n}\right), \quad \xi=\left(\xi_{1}, \xi_{2}, \ldots, \xi_{n}\right)$ are points of $\mathbb{R}^{n},<t, \xi>=t_{1} \xi_{1}+\ldots+t_{n} \xi_{n}$. For $1 \leq p \leq \infty$ we denote by $\|f\|_{p}$ the $L_{p}$-norm of a function $f$. Let $\Omega \subset \mathbb{R}^{n}$ be an arbitrary bounded domain and let $1 \leq p \leq \infty$. We denote by $L_{p, \Omega}$ the space of all functions $f$ such that the norm $\|f\|_{p}$ is finite, and the Fourier transforms $F$ of $f$ are supported in $\Omega$. Such functions $f$ vanish at infinity in $\mathbb{R}^{n}$ and can be extended into the

1991 Mathematics Subject Classification: Primary 30D10; Secondary 81B10.

The paper is in final form and no version of it will be published elsewhere. 
complex space $\mathbb{C}^{n}$ as entire functions of exponential type. In one-dimensional case we assume that $\Omega_{\sigma}=\{x:-\sigma<x<\sigma\}$ and we denote $L_{p, \Omega}=L_{p, \sigma}$.

I would like to express my sincere gratitude to Prof. J. Ławrynowicz for the possibility to lecture in this well-known mathematical Centre.

1. One-dimensional case. Let $I_{N}$ be an arbitrary interval of length $N$. For an arbitrary measurable set $M$ we denote by $|M|$ its Lebesgue measure.

DeFinition 1. We define the asymptotical density $\beta(M)$ of an arbitrary measurable set $M$ as

$$
\beta(M)=\varlimsup_{N \rightarrow \infty} \inf \left|M \cap I_{N}\right| / N
$$

DeFinition 2. We define

$$
\widetilde{\gamma}(M)=\inf \left\{N: \inf \left|M \cap I_{N}\right|=N \beta(M) / 2\right\} .
$$

It is obvious that the necessary condition for the estimate under consideration

$$
\|w f\|_{p} \geq \mathrm{K}(\sigma)\|f\|_{p}, \quad f \in L_{p, \sigma}
$$

to be valid with $\mathrm{K}(\sigma) \rightarrow \infty$ as $\sigma \rightarrow 0$, is $\varlimsup_{|t| \rightarrow \infty} w(t)=\infty$. Therefore, from now on this condition is assumed to hold.

For an arbitrary continuous function $w$ and for $\tau>0$ put $M_{\tau}^{w}=\{t:|w(t)|>\tau\}$ and denote $\widetilde{\gamma}_{w}(\tau)=\widetilde{\gamma}\left(M_{\tau}^{w}\right)$. It is clear that $\varlimsup \widetilde{\gamma}_{w}(\tau)=\infty$ as $\tau \rightarrow \infty$ because $\overline{\lim } w(t)=\infty$.

DEFINITION 3. Let $\gamma_{w}(\tau)$ be the least left semicontinuous nondecreasing majorant for $\widetilde{\gamma}_{w}(\tau)$. We define the nondecreasing function

$$
\Gamma_{w}(\lambda)=\inf \left\{\tau: \gamma_{w}(\tau) \geq \lambda\right\} .
$$

It is obvious that for and arbitrary $\lambda>0$ we have $\left.\left(\gamma_{w}\right) \circ \Gamma_{w}\right)(\lambda) \leq \lambda$ and $\left(\gamma_{w} \circ \Gamma_{w}\right)(\lambda)=$ $\lambda$ if the function $\Gamma_{w}$ is continuous and increases at the point $\lambda$. One can regard the function $\Gamma_{w}$ as the right inverse function to $\gamma_{w}$.

Example. Let $w:[0, \infty) \rightarrow[0, \infty)$ be an increasing function for which $w(\infty)=\infty$. Then $\beta\left(M_{\tau}^{w}\right)=1$ for every $\tau>0$ and $\gamma_{w}(\tau)=2 w^{-1}(\tau)$. (Here and later $G^{-1}$ denotes the inverse function to $G)$. Thus, in this case $\Gamma_{w}(\lambda)=w(\lambda / 2)$.

Now we can formulate one of the main results of this work.

THEOREM 1. Let $w$ be an arbitrary continuous function such that

$$
\beta\left(M_{\tau}^{w}\right) \geq \beta_{0}>0
$$

for all sufficiently large $\tau$. Then there is a constant $c>0$ which does not depend on $f$ or on $\sigma$ such that for all $p, 1 \leq p \leq \infty$, the estimate

$$
\|w f\|_{p} \geq c \Gamma_{w}\left(\sigma^{-1}\right)\|f\|_{p}, \quad f \in L_{p, \sigma}
$$

is valid. 
COROLlaRY 1. If $\Psi(t)$ is an increasing function and $\gamma_{w}(t) \leq \Psi(t)$ for all sufficiently large $t>t_{0}$ then the estimate

$$
\|w f\|_{p} \geq c \Psi^{-1}\left(\sigma^{-1}\right)\|f\|_{p}, \quad f \in L_{p, \sigma}
$$

holds. Here a constant $c$ does not depend either on $\sigma$ or $f$.

Our next result is related to sharp estimates of the described type. Let us start with a definition.

Definition 4. We say that the estimate

$$
\|w f\| \geq c \mathrm{~K}(\sigma)\|f\|_{p}, \quad f \in L_{p . \sigma}
$$

is $\operatorname{sharp}($ as $\sigma \rightarrow 0)$ if any other estimate of the same type

$$
\|w f\| \geq c \mathrm{~K}_{1}(\sigma)\|f\|_{p}, \quad f \in L_{p, \sigma}
$$

implies the inequality $\mathrm{K}_{1}(\sigma) \leq c \mathrm{~K}(\sigma)$ for all $\sigma>0$.

One of the typical estimates of this kind is the well-known Hardy's inequality

$$
\|t f\|_{2} \geq(2 \sigma)^{-1}\|f\|_{2}, \quad f \in L_{2, \sigma} .
$$

TheOREm 2. Assume that for all sufficiently large $t$ and $\lambda, \quad t \geq A, \quad \lambda \geq B$, there is such a number $N$ that

$$
w(t \lambda) \leq \Gamma_{w}(\lambda) t^{N}
$$

Then the estimate (1) is sharp.

COROLlary 2. If for a function $\psi$ from Corollary 1 the double inequality $c \Psi(t) \leq$ $\gamma_{w}(t) \leq \Psi(t)$ holds for all sufficiently large $t>t_{0}$ with a constant $c$, which does not depend on $t$, then the estimate (2) is sharp.

Example. Let $P$ be an arbitrary nonzero complex valued polynomial of the degree $N, \quad \alpha \geq 0, \quad \beta \geq 1, \quad 1 \leq p \leq \infty$. Then, according to Theorem 1,

$$
\left\|P^{\alpha}(t) \sin \left(t^{\beta}\right) f(t)\right\|_{p} \geq c \sigma^{-\alpha N}\|f\|_{p}, \quad f \in L_{p, \sigma}
$$

and this estimate is sharp by Theorem 2 .

The following proposition allows us to obtain new weight functions $w$ of the considered type if one such function is already available.

THEOREM 3. Assume that for all $t$ larger than some constant $T \geq 0$, and for an arbitrary constant $B>0$ the function $\phi$ satisfies the conditions

$$
\phi \prime(t) \geq r>0, \quad 0<r_{1}(B) \leq \phi \prime(t+B) / \phi \prime(B) \leq r_{2}(B)<\infty
$$

If the function $w$ satisfies the condition of Theorem 1 then the estimate

$$
\|(w \circ \phi) f\|_{p}>c\left(\Gamma_{w} \circ \phi\right)\left(\sigma^{-1}\right)\|f\|_{p}, \quad f \in L_{p, \sigma}
$$

is valid.

Let us note that the condition $\phi^{\prime}(t) \geq r>0$ is essential. For instance if

$$
w=|t|^{\alpha} \sin |t|, \quad \alpha<1 ; \quad \phi=|t|^{1 / 2}
$$


then both $w$ and $\phi$ are weighted functions of the considered type and generate sharp estimates

$$
\|w f\|_{p} \geq c \sigma^{-\alpha}\|f\|_{p}, \quad\|w f\|_{p} \geq c \sigma^{-1 / 2}\|f\|_{p}
$$

for all $f \in L_{p, \sigma}$. But for the composite function $W=w \circ \phi$ the estimate $\|W f\|_{p} \geq$ $c\|f\|_{p}, \quad f \in L_{p, \sigma}$ can be valid only when $c=0$.

2. Multidimensional case. Consider a bounded convex domain $\Omega \subset \mathbb{R}^{n}, \quad 0 \in \Omega$ with the support function

$$
\mathcal{H}_{\Omega}(\tau)=\sup _{t \in \Omega}<t, \tau>
$$

For an arbitrary unit vector $\tau \in \mathbb{R}^{n}, \quad|\tau|=1$, we denote by $\delta_{\tau}(\Omega)$ the width of the domain $\Omega$ in the direction $\tau$, i.e. $\delta_{\tau}(\Omega)=\mathcal{H}_{\Omega}(\tau)+\mathcal{H}_{\Omega}(-\tau)$. In addition to this notation, for an arbitrary measurable set $U \subset \mathbb{R}^{n}$ and unit vector $\tau \in \mathbb{R}^{n}$ we denote by $d_{\tau}(U)$ the diameter of the set $U$ in the direction $\tau$. In other words

$$
d_{\tau}(U)=\sup _{\xi \in U}\left|\left\{t \in \mathbb{R}^{1}: \xi+t \tau \in U\right\}\right| .
$$

Let $\mathfrak{N}$ be the Stiefel manifold of all orthonormal bases $\mathrm{w}=\left\{w_{1}, w_{2}, \ldots, w_{n}\right\}$ in the space $\mathbb{R}^{n}$. Put

$$
\delta_{\mathrm{w}}(\Omega)=\left\{\delta_{\mathrm{w}_{1}}(\Omega), \delta_{\mathrm{w}_{2}}(\Omega), \ldots, \delta_{\mathrm{w}_{n}}(\Omega)\right\}
$$

and for an arbitrary $\sigma=\left(\sigma_{1}, \sigma_{2}, \ldots, \sigma_{n}\right) \in \mathbb{R}^{n}$ put

$$
\delta_{\mathrm{w}}^{-\sigma}(\Omega)=\delta_{\mathrm{w}_{1}}^{-\sigma_{1}}(\Omega) \delta_{\mathrm{w}_{2}}^{-\sigma_{2}}(\Omega) \ldots \delta_{\mathrm{w}_{n}}^{-\sigma_{n}}(\Omega)
$$

We denote by $\alpha=\left(\alpha_{1}, \alpha_{2}, \ldots \alpha_{n}\right)$ an arbitrary multi-index of nonnegative integers $\alpha_{j}$ with the lenght $|\alpha|=\alpha_{1}+\alpha_{2}+\ldots+\alpha_{n}$.

For arbitrary $\xi \in \mathbb{R}^{n}$ we put

$$
\xi^{\alpha}=\xi_{1}^{\alpha_{1}} \xi_{2}^{\alpha_{2}} \ldots \xi_{n}^{\alpha_{n}}
$$

Further, for any nonnegative n-tuple $\delta=\left(\delta_{1}, \delta_{2}, \ldots, \delta_{n}\right)$ we set

$$
(\delta / \alpha)^{\alpha}=\left(\delta_{1} / \alpha\right)^{\alpha_{1}}\left(\delta_{2} / \alpha_{2}\right)^{\alpha_{2}} \ldots\left(\delta_{n} / \alpha_{n}\right)_{n}^{\alpha}
$$

where the factor $\left(\delta_{j} / \alpha_{j}\right)^{\alpha_{j}}$ is omitted if $\alpha_{j}=0$.

An arbitrary polynomial $P(\xi)=P\left(\xi_{1}, \xi_{2}, \ldots, \xi_{n}\right)$ of degree $\mathcal{M}$ may be written in the form $P(\xi)=\sum_{|\alpha| \leq m} a_{\alpha} \xi^{\alpha}$, where $a_{\alpha} \neq 0$ for at least one multi-index $\alpha$ with $|\alpha|:=\alpha_{1}+\alpha_{2}+\ldots+\alpha_{n}=m$.

For every unit vector $\tau \in \mathbb{R}^{n}$ let $\partial_{\tau} P(\xi)$ denote the derivative of $P(\xi)$ in the direction of $\tau$. If $\mathrm{w} \in \mathfrak{N}$ is one of the orthonormal bases in $\mathbb{R}^{n}$ we put

$$
\partial_{\mathrm{w}}^{\alpha} P=\partial_{w_{1}}^{\alpha_{1}} \partial_{w_{2}}^{\alpha_{2}} \ldots \partial_{w_{n}}^{\alpha_{n}} P
$$

The following definition plays an important role what follows.

Definition 5. Given a polynomial $P(\xi)=\sum a_{\alpha} \xi^{\alpha}$, we call a multi-index $\alpha=$ $\left(\alpha_{1}, \alpha_{2}, \ldots, \alpha_{n}\right)$ a leading multi-index of $P(\xi)$ with respect to a basis w $\in \mathfrak{N}$ if $\partial_{\mathrm{w}}^{\alpha} P(\xi) \equiv$ const $\neq 0$ and $\partial_{w_{1}}^{\alpha_{1}} \partial_{w_{2}}^{\alpha_{2}} \ldots \partial_{w_{j}}^{\alpha_{j}+1} P(\xi) \equiv 0$ for all $j=1,2, \ldots, n$ such that $\alpha_{j} \neq 0$. 
The set of all leading multi-indeces of $P(\xi)$ with respect to a basis w will be denoted by $\mathfrak{A}_{\mathrm{w}}(P)$. Let us introduce the constant

$$
\mathrm{K}_{P}(\Omega)=\sup _{\substack{\mathrm{w} \in \mathfrak{N} \\ \alpha \in \mathfrak{A}_{\mathrm{w}}(P)}} \delta_{\mathrm{w}}^{-\alpha}(\Omega)\left|\partial_{\mathrm{w}}^{\alpha} P\right|
$$

The following theorem contains the main multi-dimensional result of the paper.

TheOREM 3. Let $\Phi:[0, \infty) \rightarrow[0, \infty)$ be an arbitrary nondecreasing function and let $P(\xi)$ be an arbitrary complex valued polynomial of degree $m$. Then for every $p \geq 1$ there exists a constant $c=c(p, n, m)$ such that for all function $u \in \mathrm{L}_{p, \Omega}$ the inequality

$$
\|\Phi(|P|) f\|_{p} \geq c \Phi\left(\mathrm{K}_{P}(\Omega)\right)\|f\|_{p} .
$$

holds for all functions $f \in E_{p, \Omega}$. If $\Phi(\infty)=\infty$ and $\partial_{\tau} P \not \equiv 0$ for every vector $\tau \neq 0$, then $\Phi\left(\mathrm{K}_{P}(\Omega)\right) \rightarrow \infty$ as the domain $\Omega$ shrinks.

Rem arks. The condition $\partial_{\tau} P \not \equiv 0$ for all $\tau \neq 0$ means that the polynomial $P$ really depends on all variables.

The domain $\Omega$ shrinks if there exists a system of convex domains $\Omega_{s}$ and unit vectors $\tau(s), \quad s \geq 0$ such that $\Omega_{0}=\Omega, \quad \Omega_{s} \supset \Omega_{r}$ for $s<r$ and $\delta_{\tau(s)}\left(\Omega_{s}\right) \rightarrow 0$ as $s \rightarrow \infty$. Let us consider some particular cases of this result.

Take $p=2, \Phi(z)=z$. Than the inequality of the Theorem and Parsevals' equality give us support dependent form of the famous Hörmander's inequality for an arbitrary PDO,

$$
\|P(\mathcal{D}) F\|_{\mathrm{L}_{2}} \geq c \mathrm{~K}_{P}(\Omega)\|F\|_{\mathrm{L}_{2}}, \quad F \in \mathrm{L}_{2}(\Omega) .
$$

Take $p=2, \quad \Phi(z)=\sqrt{z}$ and $P(\xi) \geq 0$. Then (3) coincides with a support dependent form of Gårding's inequality

$$
\operatorname{Re}(P(\mathcal{D}) F, F) \geq c \mathrm{~K}_{P}(\Omega)\|F\|_{\mathrm{L}_{2}}^{2}, \quad F \in \mathrm{L}_{2}(\Omega) .
$$

Take $p=1$. Then (3) gives us a good estimate of another kind, namely,

$$
\|\Phi(|P|) F\|_{1} \geq c \Phi\left(\mathrm{K}_{P}(\Omega)\right)\|F\|_{1} \geq c \Phi\left(\mathrm{K}_{P}(\Omega)\right) \sup _{\Omega}|F|
$$

(We remind that $F$ is the Fourier transform of $f$ ).

It turns out that function $\Phi$ does not have to be nondecreasing for some estimate of the form (3) to be valid. For instance, if $\beta\left(M_{\tau}^{\Phi}\right) \geq \beta_{0}>0$, then for some constant $c>0$

$$
\|\Phi(|P|) f\|_{p} \geq c\|f\|_{p}, \quad f \in \mathrm{L}_{p, \sigma}
$$

(E. Tel, Thesis, Technion, 1994).

It will be interesting to generalize the result of Theorem 2 and to find dependence of the constant $c$ in (4) on $\Phi$ and $P$. Nothing is known about the estimate $\|w f\| \geq \mathrm{K}(\Omega)\|f\|_{p}$ for the general weighted function $w$ in the multidimensional case.

In conclusion let us point out that the first part of this paper has some intersections with my paper [1] "On sharp support-dependent wieghted norm estimates for Fourier transforms", International Mathematical Research Notices (IMNR) 11 (1993), 289-294. The proof of Theorem 3 will be published in [2] "Support dependent weighted norm estimates for Fourier transforms", J. of Math. Anal. and Appl., to appear. 